\title{
Continuous fascia iliaca block for acute hip fractures: a randomized-controlled pilot study
}

\author{
Vicky Loessin, MD (1) • A. J. EIZahabi, MD • Brian Brownbridge, MD, FRCPC • \\ Jagmeet Singh Bajwa, MSc
}

Received: 13 May 2019/Revised: 11 June 2019/Accepted: 12 June 2019/Published online: 25 June 2019

(c) Canadian Anesthesiologists' Society 2019

\section{To the Editor,}

We read with interest the letter from Henderson et al. regarding improving care for elderly patients with hip fracture through interdisciplinary collaboration in regional anesthesia. ${ }^{1}$ We recently completed a randomizedcontrolled pilot study of 30 patients looking at continuous fascia iliaca blocks (cFIBs) to improve pain in hip fracture patients waiting for surgery (clinicaltrials.gov NCT03588689).

The fascia iliaca block (FIB) is a compartment field block that targets the femoral, obturator, and the lateral cutaneous nerve of the thigh. Studies have shown the efficacy of FIBs in treating pain from hip fractures, and in surgeries involving the hip, knee, and anterior thigh. Successful blockade requires deposition of large volumes of local anesthetic $(30-40 \mathrm{~mL}){ }^{2,3}$

Because wait times in our institution average two days from the time of hip fracture until surgical repair, we hypothesized that a cFIB would improve pain control and decrease opioid use, thereby showing benefits in markers associated with perioperative morbidity-delirium, respiratory complications such as pneumonia, improved patient positioning, and decreased length of stay. ${ }^{4}$

We recruited patients from May 2018 to March 2019 at the Royal University Hospital in Saskatoon, SK. Researchers were notified when a patient aged $65 \mathrm{yr}$ or

Vicky Loessin and A. J. ElZahabi are co-first authors.

V. Loessin, MD ( $₫)$ · A. J. ElZahabi, MD · B. Brownbridge, MD, FRCPC · J. S. Bajwa, MSc

Department of Anesthesiology, Perioperative Medicine and Pain Management, University of Saskatchewan, Saskatoon, SK, Canada

e-mail: Vicky.Loessin@usask.ca older was admitted to hospital with an acute hip fracture. We excluded patients with an American Society of Anesthesiologists score $\geq 4$, open fracture, concomitant injuries that might interfere with positioning, local anesthetic allergy, delirium or cognitive impairment preventing consent, infection or previous surgery at the femoral triangle, and those using warfarin, anti-Xa inhibitors, or long-term opioids. After obtaining written informed consent, patients were randomized to receive either standard pain orders (including opioids and other adjuncts such as non-steroidal anti-inflammatory drugs and acetaminophen), or standard pain orders plus a cFIB. Patients in the cFIB group received an initial bolus of 40 $\mathrm{mL} 0.125 \%$ ropivacaine, then ropivacaine $0.2 \% \quad 10$ $\mathrm{mL} \cdot \mathrm{hr}^{-1}$ until the time of surgery. In the operating room (OR), catheters were re-bolused with $40 \mathrm{~mL} 0.125 \%$ ropivacaine then removed. Data were collected twice daily from the time of enrolment until discharge. Mortality data were collected 30 days post-discharge.

Our primary outcome measured the incidence of respiratory complications from admission to discharge. We used oxygen saturation trends, oxygen requirements, need for positive pressure ventilation, and radiographic evidence of atelectasis or pneumonia as surrogates for respiratory complication. We found no differences in respiratory outcomes between groups. Our secondary outcomes included pain scores, opioid side effects, delirium, total opioid dose, postoperative length of stay, and 30-day mortality. Patients in the cFIB group had significantly lower pain scores preoperatively compared with the standard care group in the supine, semi-recumbent positions, and with active motion (Table). We found no difference in the incidence of opioid-related side effects, delirium, total opioid dose, length of stay, or 30-day mortality. There was a higher incidence of surgery under 
Table Outcomes of a randomized-controlled trial comparing cFIB with standard care for hip fracture patients

\begin{tabular}{|c|c|c|c|}
\hline Outcome & Standard care $(n=15)$ & Standard care + cFIB $(n=15)$ & $P$ value \\
\hline Respiratory complications & $4 / 15(27 \%)$ & $4 / 15(27 \%)$ & $1.000^{\mathrm{a}}$ \\
\hline \multicolumn{4}{|l|}{ Pain scores } \\
\hline \multicolumn{4}{|l|}{ Supine } \\
\hline Pre-enrolment & $6[3-7]$ & $6[3-8]$ & $0.933^{\mathrm{c}}$ \\
\hline Post-enrolment & $6[3-7]$ & $0[0-2]$ & $<0.001^{\mathrm{c}}$ \\
\hline Preoperative & $4[2-6]$ & $1[0-2]$ & $<0.001^{\mathrm{c}}$ \\
\hline \multicolumn{4}{|l|}{ Semi-recumbent } \\
\hline Pre-enrolment & $6[3-8]$ & $5[4-7]$ & $0.557^{\mathrm{c}}$ \\
\hline Post-enrolment & $6[3-8]$ & $0[0-3]$ & $<0.001^{\mathrm{c}}$ \\
\hline Preoperative & $5[2-8]$ & $1[0-1]$ & $<0.001^{\mathrm{c}}$ \\
\hline \multicolumn{4}{|l|}{ Active } \\
\hline Pre-enrolment & 9 [8-9] & $8[5-9]$ & $0.348^{\mathrm{c}}$ \\
\hline Post-enrolment & $8[8-10]$ & $3[2-5]$ & $<0.001^{\mathrm{c}}$ \\
\hline Preoperative & $7.5[5-8]$ & $3[2-5.5]$ & $0.003^{\mathrm{c}}$ \\
\hline \multicolumn{4}{|l|}{ Opioid side effects } \\
\hline Post-enrolment & $7 / 15(47 \%)$ & $3 / 15(20 \%)$ & $0.121^{\mathrm{b}}$ \\
\hline Preoperative & $12 / 15(80 \%)$ & $9 / 15(60 \%)$ & $0.427^{\mathrm{b}}$ \\
\hline \multicolumn{4}{|l|}{ Delirium } \\
\hline Postoperative & $3 / 15(20 \%)$ & $2 / 15(13 \%)$ & $1.000^{\mathrm{a}}$ \\
\hline Total opioid up to POD \#3 & $31[6.5-69]$ & $35.5[5-61.5]$ & $0.967^{\mathrm{c}}$ \\
\hline 30-day mortality & 0 & 2 & \\
\hline
\end{tabular}

Pain scores: mean [interquartile range]. Pain scores on visual analogue scale 1-10.

Respiratory complications included pneumonia, atelectasis, supplemental oxygen, sat $<92 \%$, or requiring positive pressure ventilation. Opioidrelated side effects included nausea, vomiting, pruritis, constipation, and urinary retention. Total opioid dose was in morphine intravenous equivalents. ${ }^{\mathrm{a}}$ Fisher's exact test; ${ }^{\mathrm{b}}$ Pearson Chi square test; ${ }^{\mathrm{c}}$ Mann-Whitney test. cFIB $=$ continuous fascia iliaca block; POD $=$ postoperative day

subarachnoid block $(\mathrm{SAB})$ in the treatment group, 53\% compared with $40 \%$, perhaps because of improved tolerance of positioning in patients to facilitate SAB. The feedback we received from members of the care team was overwhelmingly positive, likely reflecting ease of caring for patients with cFIBs.

We faced many institutional barriers to performing cFIBs in these patients. Our emergency department did not permit us to perform the cFIB in the unit because they do not train their nurses to monitor regional catheters. We had to wait until patients were assigned a bed on the ward, then perform blocks in the OR recovery room, which resulted on average in a one-day delay between fracture and placement of cFIB. Additionally, since many of these patients are admitted at night, we would need in-house personnel such as emergency physicians or anesthesia residents to perform the blocks in a timely manner if we were to implement cFIBs for hip fracture patients.

We had aimed to recruit 50 patients, but stopped at 30 because of challenges in recruiting patients. Although these institutional barriers did not help, our recruitment was most significantly hampered by the extremely large fraction of these patients who had dementia and who were unable to consent to participate in the study. Excluding these patients likely limits the generalizability of our results to the hip fracture population as a whole.

Providing cFIBs is a value-added service for our patients, especially considering the average time of two days from fracture to surgical repair in our institution. We are hopeful that we can use the multidisciplinary approach and pathway from Henderson and colleagues to work towards implementing change in how we care for elderly patients with acute hip fractures in our institution.

Conflicts of interest None declared.

Editorial responsibility This submission was handled by Dr. Philip M. Jones, Associate Editor, Canadian Journal of Anesthesia.

\section{References}

1. Henderson $C Y$, Abdel-Galil R, Woo MY, et al. Improving care for elderly patients with hip fracture: interdisciplinary collaboration in regional analgesia. Can J Anesth 2019; DOI: https://doi.org/10. 1007/s12630-019-01362-3. 
2. Foss NB, Kristensen BB, Bundgaard $M$, et al. Fascia iliaca compartment blockade for acute pain control in hip fracture patients: a randomized, placebo-controlled study. Anesthesiology 2007; 106: 773-8.

3. Wambold $D$, Carter $C$, Rosenberg $A D$. The fascia iliaca block for postoperative pain relief after knee surgery. Pain Pract 2001; 1 : 274-7.
4. Guay J, Parker MJ, Griffiths R, Kopp S. Peripheral nerve blocks for hip fractures. Cochrane Database Syst Rev 2017; 5: CD001159.

Publisher's Note Springer Nature remains neutral with regard to jurisdictional claims in published maps and institutional affiliations. 